\title{
Vegetação lenhosa de regiões semiáridas em diferentes altitudes
}

\author{
Woody vegetation of semi-arid regions at different altitudes
}

\begin{abstract}
Wedson Batista dos Santos', Luiz Carlos Marangon", Fernando José Freire"II, Rafael Leite Braz ${ }^{\mathrm{II}}$, José Edson de Lima Torres ${ }^{\mathrm{I}}$, José Nailson Barros Santos ${ }^{\mathrm{IV}}$
\end{abstract}

\begin{abstract}
Resumo
O objetivo deste trabalho foi avaliar diversidade florística em diferentes altitudes na região do Araripe em Pernambuco, Brasil. Metodologicamente, a região foi estratificada em três níveis de altitude: inferior (até $600 \mathrm{~m}$ ), intermediário (maior que 600 e menor que $750 \mathrm{~m}$ ) e superior (igual ou maior que $750 \mathrm{~m}$ ). Foram inventariados nove fragmentos florestais, três por estrato altitudinal. Em cada fragmento, distribuíramse 20 unidades amostrais retangulares, de $250 \mathrm{~m}^{2}$ cada, totalizando área amostral de 4,5 ha. Nas áreas amostrais foram quantificados, coletados e identificados todos os indivíduos lenhosos com nível de inclusão de circunferências a altura do peito (CAP) maior ou igual a $0,10 \mathrm{~m}$ (CAP a 1,30 $\mathrm{m} \geq 0,10 \mathrm{~m}$ ). Analisou-se, riqueza e similaridade florística entre fragmentos e níveis de altitudes. Foram amostrados 6.987 indivíduos, distribuídos em 35 famílias botânica, 82 gêneros e 153 espécies. A região apresenta grande riqueza florística, com variação entre e dentro dos três níveis altitudinais, com tendência de aumento na diversidade com a elevação da altitude. A maioria das espécies apresentaram pouca plasticidade fenotípica, com ocorrência e abundância restrita ao fragmento ou altitude. Em pequenas distâncias geográficas foram diagnosticadas relevantes variações fitofisionômicas, o que corrobora a importância da estratificação de altitudes e replicação de fragmentos na amostragem. Pelo nível de degradação antropogênica e a intensificação das atividades florestais da região, faz-se necessário e urgente a criação de políticas públicas voltadas para práticas sustentáveis no uso da floresta e seu processo de conservação. Para tanto, o negligenciamento a esta problemática, coloca em risco de extinção uma grande representação da diversidade vegetacional do nordeste brasileiro.
\end{abstract}

Palavras-chave: Floresta seca; Caatinga; Florística

Engenheiro Florestal, Dr., Departamento de Ciência Florestal, Universidade Federal Rural de Pernambuco, Rua Dom Manuel de Medeiros, s/n, Dois Irmãos, CEP 52171-900, Recife (PE), Brasil. wedsonfl@hotmail.com (ORCID: 0000-0002-2598-1971)/ edsonfloresta@yahoo.com.br (ORCID: 0000-0002-6844-1393)

II Engenheiro Florestal, Dr., Professor do Departamento de Ciência Florestal, Universidade Federal Rural de Pernambuco, Rua Dom Manuel de Medeiros, s/n, Dois Irmãos, CEP 52171-900, Recife (PE), Brasil. lcmarang@terra.com.br (ORCID: 0000-0002-5663-0381) / rlbraz.ufrpe@gmail.com (ORCID: 0000-0001-8473-3233)

III Engenheiro Agrônomo, Dr., Professor do Departamento de Agronomia, Universidade Federal Rural de Pernambuco, Rua Dom Manuel de Medeiros, s/n, Dois Irmãos, CEP 52171-900, Recife (PE), Brasil. fernandojfreire@uol.com.br (ORCID: 0000-0002-3264-712X)

IV Graduado em Ciências Agrárias, Mestre em Ciências Florestais, Departamento de Ciência Florestal, Universidade Federal Rural de Pernambuco, Rua Dom Manuel de Medeiros, s/n, Dois Irmãos, CEP 52171-900, Recife (PE), Brasil. nailson.gba@hotmail.com (ORCID: 0000-0003-4348-3789) 


\begin{abstract}
This work aimed to evaluate the floristic diversity at different altitudes in the Araripe region of Pernambuco state, Brazil. For method purposes, the region was stratified into three levels of altitude: inferior (until 600 $\mathrm{m})$, intermediate $(>600$ and $<750)$ and superior $(\geq 750 \mathrm{~m})$. Nine forest fragments were inventoried, three by altitudinal stratum. Twenty rectangular sampling units of $250 \mathrm{~m}^{2}$ each were distributed for each fragment, totaling a sampling area of 4.5 ha. All woody individual's quantification, collection, and identification were performed with respect to the Circumference at Breast Height $(\mathrm{CBH})$ greater than or equal to $0.10 \mathrm{~m}$ $(\mathrm{CBH}$ at $1.30 \mathrm{~m} \geq 0.10 \mathrm{~m})$. The richness and the floristic similarity among the fragments and altitude levels were analyzed. A total of 6,987 individuals were sampled and distributed in 35 botanical families, 82 genera and 153 species. The region presented great floristic richness, with variation among and within the three altitudinal levels and the diversity demonstrated to increase with the altitude. Most species exhibited little phenotypic plasticity, with occurrence and abundance restricted to the fragment or the altitude level. At short geographical distances, relevant phyto-physiognomic variations were diagnosed, which corroborates with the importance of altitude stratification and fragment replication in the sampling procedures. Due to the level of anthropogenic degradation and the intensification of forest activities in the region, it is, therefore, necessary and urgent to create public policies aiming sustainable practices in forest use and its conservation process. To this end, the negligence of this problem puts at risk of extinction a large representation of the Brazilian northeastern vegetation diversity.
\end{abstract}

Keywords: Dry forest; Caatinga; Floristics

\title{
Introdução
}

A região semiárida do Nordeste tem sofrido um dos maiores impactos ambientais dos últimos anos, principalmente, por abrigar uma grande concentração de empresas extratoras de minérios, em que se destaca o Polo Gesseiro do Araripe, o maior produtor de gesso do Brasil, responsável por aproximadamente, $95 \%$ de toda produção nacional. Esse setor utiliza como principal fonte de energia a biomassa florestal, resultando em elevada pressão antrópica sobre a vegetação nativa, que vem sendo devastada, ocasionando em muitas áreas degradadas, desertificadas e/ou em processo de desertificação. O problema se agrava por essa região ser circundada por serras, com variados efeitos orográficos, responsáveis pela formação de distintos micro-hábitats com elevada diversidade biológica (ARAÚJO et al., 2011; GADELHA et al., 2015).

A vegetação de regiões semiáridas geralmente é caracterizada pela ausência de folhas na maior parte do ano, mecanismo de sobrevivência ao clima seco, com baixos volumes de precipitação e elevadas temperaturas. $\mathrm{O}$ aspecto seco, muitas vezes, gera uma interpretação equivocada sobre variabilidade florística, levando muitos pesquisadores a generalizar a classificação e diversificação desses ambientes. Em pequenas distâncias geográficas podem ocorrer variações ambientais, responsáveis pela formação de micro-hábitats com diferentes composições florísticas. A topografia é uma variável física extremamente influenciadora na heterogeneidade ambiental, visto que, pode exercer inúmeros efeitos orográficos, alterando a dinâmica de distribuição e intensidade pluviométrica, com níveis de precipitações variados em resposta aos obstáculos topográficos (ARRUDA et al., 2015; HIGUCHI et al., 2016; NEVES et al., 2016; SANTOS; NASCIMENTO, 2017).

Os inventários florestais são extremamente eficazes no diagnóstico de possíveis variações ambientais, visto que, diferentes composições florísticas podem indicar alterações climáticas, edáficas e/ou geográficas. O clima, quando associado a tipos de solos, pode resultar em uma a flora regional descontínua, com distintos grupos de vegetação. A fitofisionomia é resultado de processos biológicos em combinação com variáveis ambientais, podendo esta ser indicadora de climas particulares, regiões biogeográficas e altitudes (ARAÚJO et al., 2011; ARAÚJO, 2011; AMJAD et al., 2016). 
O conhecimento da composição florística permite analisar semelhanças com áreas adjacentes, podendo ser determinante, para criação de políticas públicas de conservação biológica e aplicação de técnicas de manejos florestais sustentáveis (SANTANA et al., 2016; SILVA et al., 2017).

Apesar de estar evidente a importância dos efeitos orográficos sobre a variação da vegetação, em regiões semiáridas são praticamente escassos os trabalhos científicos com essa abordagem, sendo a maioria dos estudos isolados, em nível de fragmento. Partindo da hipótese que mesmo em regiões com clima semiárido, ocorre significativa variação da composição florística em função ao gradiente topográfico, o principal objetivo do trabalho foi diagnosticar a vegetação lenhosa em diferentes altitudes da região do Araripe, Pernambuco, Brasil.

\section{Material e métodos}

\section{Área de estudo: Região do Araripe, Pernambuco, Brasil}

O estudo foi desenvolvido em diferentes altitudes da região do Polo Gesseiro do Araripe, extremo oeste de Pernambuco, Brasil.

As altitudes variam entre 400 e 1000 m. O clima local é o BSh' segundo a classificação de Köppen (ALVARES et al., 2013), caracterizado como clima das estepes quentes de baixa latitude e altitude ou clima semiárido quente. Com precipitações baixas e irregulares em toda região, geralmente aumentando em elevadas altitudes. De maneira geral, a vegetação é classificada como Savana Estépica, contudo, são encontradas subdivisões fitofisionômicas como Caatinga Arbustiva, Caatinga Arbustivo-arbórea, Caatinga Arbórea, Cerrado, Cerradão, Mata Úmida, Mata Secundária, Carrasco, Contato Cerradão-carrasco e Mata Seca (BRASIL, 2007; IBGE, 2012).

Foram inventariados nove fragmentos florestais, em avançados estágios sucessionais, distribuídos em três níveis de altitudes, sendo:

Os fragmentos "F1", "F2" e "F3", pertencentes ao nível inferior de altitude (Altitude $\leq 600$ m), em que: F1 (Altitude de $540 \mathrm{~m}$ e coordenada geográfica $7^{\circ} 46^{\prime} 58.28^{\prime}$ ” ; 40²7’13.73”O), com fitofisionomia Caatinga Arbustivo-Arbórea, com indivíduos lenhosos de porte intermediário de altura $( \pm 5 \mathrm{~m})$; F2 (Altitude de $605 \mathrm{~m}$ e coordenada geográfica $7^{\circ} 36^{\prime} 19.62$ ”s; 40²8’53.67”O), vegetação Caatinga Arbórea, com porte mais elevado $( \pm 8 \mathrm{~m})$, localizado em área aparentemente mais úmida; e F3 (Altitude de $546 \mathrm{~m}$ e coordenada geográfica $7^{\circ} 42^{\prime} 02.67$ ”; $40^{\circ} 23^{\prime} 15.11^{\prime}$ 'O), fitofisionomia Caatinga Arbustivo-Arbórea com porte intermediário. Apesar de todas as áreas serem consideradas em avançados estágios sucessionais, F3 é considerada a mais jovem, visto que, já sofreu corte raso há aproximadamente 25 anos.

As áreas "F4", "F5" e "F6", fazem parte do nível intermediário de altitude $(600<$ altitude $<750$ $\mathrm{m})$, em que: as três de vegetação caducifólia com transição Caatinga-Cerrado, indivíduos lenhosos apresentando porte intermediário de altura. F4 (Altitude de $681 \mathrm{~m}$ e coordenada geográfica $7^{\circ} 46^{\prime} 58.28^{\prime}$ 'S $40^{\circ} 27^{\prime} 13.73$ ”O), com terreno inclinado, solo arenoso e cursos de água intermitente em seu interior, sendo localizada próximo ao reservatório de água; F5 (Altitude de 664 m e coordenada geográfica $7^{\circ} 31^{\prime} 09.16^{\prime \prime}$; $40^{\circ} 30^{\prime} 54.01$ ”'O), com terreno plano e solo arenoso; e F6 (Altitude de 715 m e coordenada geográfica $7^{\circ} 33^{\prime 2} 23.78$ ”s; 40³3’15.75”O), área mais seca, com terreno inclinado, solo pedregoso e com afloramento rochoso.

Os fragmentos "F7", "F8" e "F9", pertencem ao nível superior de altitude (altitudes $\geq 750$ m), onde: F7 (Altitude de 847 m e coordenada geográfica $7^{\circ} 25^{\prime} 36.28^{\prime \prime}$; $40^{\circ} 25^{\prime} 44.71$ ”O), com fitofisionomia Carrasco, vegetação caducifólia seca, com indivíduos de pequeno porte de altura $( \pm 4 \mathrm{~m}$ ), com presença de muitas lianas, terreno plano e solo arenoso; F8 (Altitude de $801 \mathrm{~m} \mathrm{e}$ coordenada geográfica $7^{\circ} 32$ ’54.81”s; 40¹8’15.98”O), com fitofisionomia de transição (Carrasco- 
Floresta Úmida), vegetação semicaducifólia, de porte intermediário de altura, terreno plano e solo arenoso; e F9 (Altitude de 951 m e coordenada geográfica $7^{\circ} 28^{\prime} 14.16$ ”'s; 39³9’38.15”O), com fitofisionomia de Floresta úmida, vegetação perenifólia de porte elevado $( \pm 8 \mathrm{~m})$, terreno plano, solo arenoso e próximo às nascentes e corpos d'água.

\section{Levantamento da vegetação Arbustiva-Arbórea e análise de dados}

Para a amostragem, foram distribuídas 180 unidades amostrais retangulares com dimensões de $250 \mathrm{~m}^{2}$ cada, totalizando uma área amostral de 4,5 ha. Para maior representatividade da região, houve estratificação em três níveis de altitudes, inferior (altitudes $\leq 600 \mathrm{~m}$ ), intermediário $(600<$ altitude $<750 \mathrm{~m})$ e superior (altitudes $\geq 750 \mathrm{~m})$. Em cada estrato foram alocadas 60 parcelas, distribuídas em três fragmentos florestais diferentes, sendo 20 em cada, somando uma área amostral de 1,5 ha por nível de altitude. As unidades amostrais foram distribuídas de forma sistemática, formando uma malha, na qual as parcelas ficaram equidistantes 25 metros umas das outras.

Dentro das unidades amostrais, foram realizadas coletas e triagens do material botânico de todos os indivíduos lenhosos com circunferência a altura do peito maior ou igual a $0,10 \mathrm{~m}$ (CAP $1,30 \mathrm{~m}$ do solo $\geq 0,10 \mathrm{~m}$ ). A identificação das espécies foi realizada com ajuda de especialistas e por meio de comparação com exsicatas depositadas no Herbário Sérgio Tavares, do Departamento de Ciência Florestal da Universidade Federal Rural de Pernambuco (HST/UFRPE), no qual foram devidamente registradas e incorporadas ao acervo.

A comparação de riqueza entre comunidades foi realizada segundo: surgimento de novas espécies, pelas curvas de rarefação, com intervalos de confiança com $95 \%$ de confiabilidade, com 1.000 randomização, utilizando o software EstimateS 9.1.0 (COLWELL, 2013); e, considerando a densidade de indivíduos por espécies, pelo teste $t$, buscando verificar possíveis semelhanças no padrão de distribuição dos índices de Shannon $\left(H^{\prime}\right)$ pelo software Past 3.2 (HAMMER; HARPER; RYAN, 2001).

Para averiguar a similaridade da composição florística entre fragmentos, foram utilizadas análises de dendrogramas elaborados sob duas perspectivas: presença e ausência, pelo método de grupos de pares não ponderados, usando médias aritméticas (UPGMA), para os índices de similaridade de Sorenson $\left(\mathrm{SO}_{i}\right)$ e Jacard; e, considerando a abundância de indivíduos, pelo método de agrupamento UPGMA e índice de similaridade de Morisita-Horn $\left(\mathrm{CH}_{\mathrm{i}}\right)$. Foi utilizada a correlação cofenética para verificar se o método de agrupamento foi satisfatório e se houve um bom grau de ajuste entre a matriz de similaridade, com a matriz resultante da simplificação. A determinação do ponto de corte dos dendrogramas para definição de grupos foi realizada conforme o método proposto por Mojena (1977).

\section{Resultados e discussões}

Foram amostrados 6.987 indivíduos, distribuídos em 35 famílias botânica, 82 gêneros e 153 espécies, sendo dessas, 16 identificadas em nível de gênero, 12 de família e 14 indeterminadas. $\mathrm{Na}$ Tabela 1, estão quantificados detalhadamente, por fragmento e para o total da amostragem na região do Araripe. 


\section{Tabela 1 - Composição florística de indivíduos lenhosos, de nove fragmentos florestais distribuídos em três níveis de altitudes na região do Araripe, Pernambuco, Brasil}

Table 1 - Floristic composition of woody individuals, from nine forest fragments distributed in three levels of altitude in the Araripe region, Pernambuco state, Brazil

\begin{tabular}{cccccc}
\hline Áreas & Indivíduos & Famílias & Gêneros & Espécies & Indeterminadas \\
\hline F1 & 436,0 & 16 & 30 & 35 & 0 \\
F2 & 1.068 & 24 & 42 & 51 & 2 \\
F3 & 553,0 & 14 & 23 & 27 & 2 \\
\hline F4 & 1.032 & 15 & 31 & 43 & 0 \\
F5 & 844,0 & 11 & 22 & 28 & 0 \\
F6 & 657,0 & 13 & 23 & 30 & 2 \\
F7 & 644,0 & 14 & 22 & 27 & 2 \\
F8 & 707,0 & 16 & 28 & 41 & 4 \\
F9 & 1.046 & 23 & 30 & 40 & 5 \\
Total & 6.987 & $35^{*}$ & $82^{*}$ & $153^{*}$ & $14^{*}$ \\
\hline
\end{tabular}

Fonte: Autores (2019)

Em que: * = Quantificação da variável em toda a amostragem, não representando o somatório das áreas, visto que, a mesma taxa pode ocorrer simultaneamente, em mais de um fragmento florestal.

\section{Similaridade entre fragmentos florestais pela distribuição de famílias botânicas}

Considerando a presença-ausência de famílias entre fragmentos, no dendrograma, verifica-se a formação de dois grupos com similaridades. O maior, com aproximadamente, $48 \%$ de similaridade, é composto por F1, F2, F3 e F4, fragmentos com altitudes de até $680 \mathrm{~m}$, que juntos compartilham 10 famílias botânicas (Anacardiaceae, Annonaceae, Capparaceae, Combretaceae, Euphorbiaceae, Fabaceae, Myrtaceae, Nyctaginaceae, Salicaceae e Sapindaceae). F5, com altitude de $664 \mathrm{~m}$, apesar de pelo ponto de corte não pertencer ao "grupo 1", apresentou boa similaridade com este, possuindo oito famílias comuns, com ausência de Salicaceae e Sapindaceae.

O segundo grupo, formado por F6, F7 e F8, fragmentos com altitudes superiores a $700 \mathrm{~m}$ e localizados em regiões semiáridas, apresentaram sete famílias compartilhadas (Annonaceae, Combretaceae, Euphorbiaceae, Fabaceae, Myrtaceae, Nyctaginaceae e Rutaceae).

O fragmento $\mathrm{F} 9$, que possui a maior altitude $(951 \mathrm{~m})$ e clima mais úmido, apresentou baixa similaridade aos dos demais, com oito famílias de ocorrência exclusiva (Hypericaceae, Lauraceae, Melastomataceae, Ochnaceae, Proteaceae, Simaroubaceae, Chrysobalanaceae e Sapotaceae) (Figura 1). 
Figura 1 - Dendrograma de agrupamento pelo método de grupo (UPGMA), com base no coeficiente de Jacard e na variável família botânica, para nove fragmentos florestais distribuídos em três níveis de altitudes na região do Araripe, Pernambuco, Brasil. Sendo: CCC, coeficiente de correlação cofenética; e $M$, ponto de corte conforme o índice de Mojena (1977)

Figure 1 - Group dendrogram by group method (UPGMA), based on the Jaccard index and the botanical family variable, for nine forest fragments distributed in three altitude levels in the Araripe region, Pernambuco state, Brazil. Being: CCC, co-behavioral correlation coefficient; and $M$, cut-off point according to the Mojena index (1977)

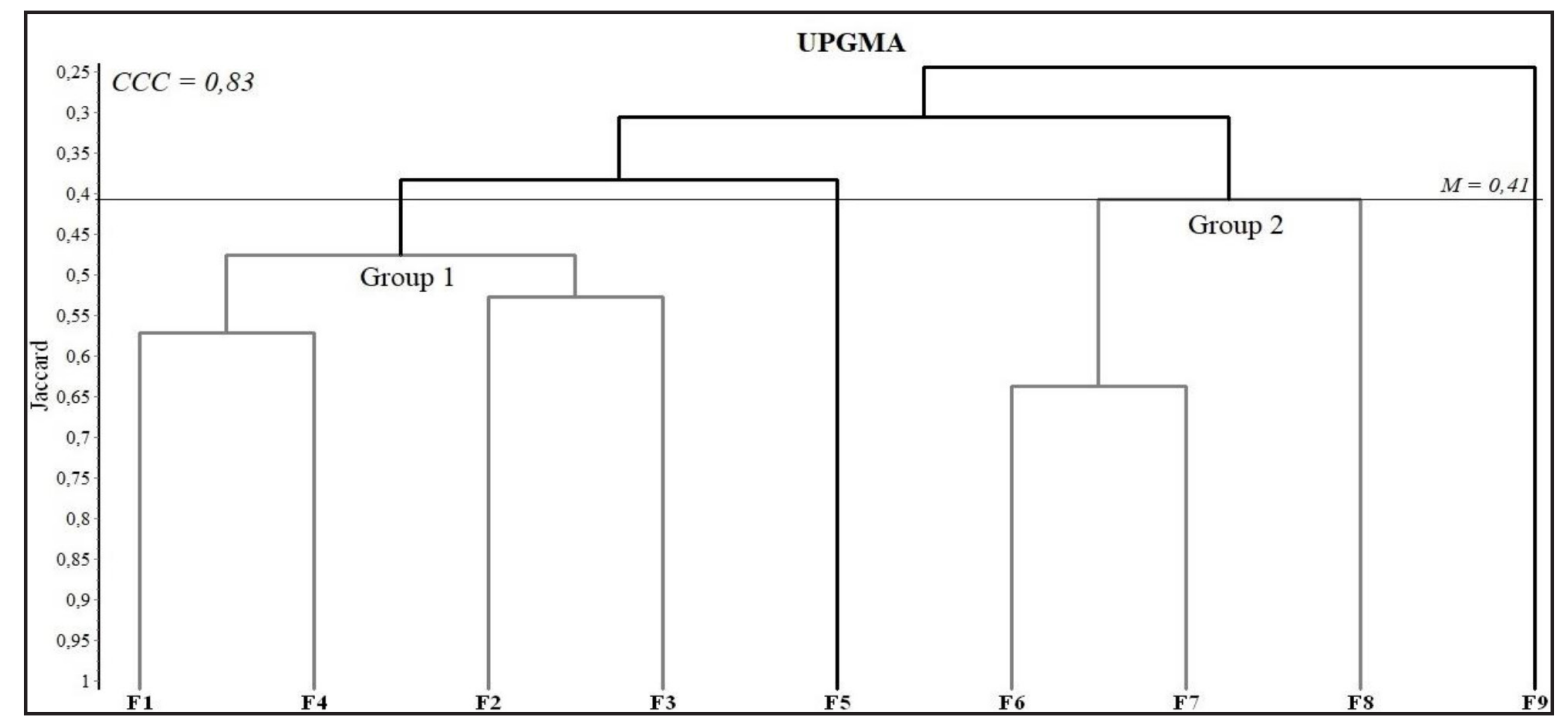

Fonte: Autores (2019)

Considerando a abundância, as 10 famílias com maiores números de indivíduos na região foram: Fabaceae, com 2.015, Euphorbiaceae, com 1.239, Combretaceae, com 505, Rutaceae, com 367, Nyctaginaceae, com 321, Lauraceae e Myrtaceae, com 251, Capparaceae, com 233, Sapindaceae, com 216 e Anacardiaceae com 198, representando estas, aproximadamente, 80\% do total de indivíduos da amostragem.

Nos fragmentos do nível inferior de altitude $(\leq 600 \mathrm{~m})$, ocorreu maior concentração de indivíduos em duas famílias, Fabaceae e Euphorbiaceae, representando estas, $78 \%$ do total de F1, $51 \%$ de $\mathrm{F} 2$ e $68 \%$ de $\mathrm{F} 3$.

No nível intermediário de altitudes (de 600 a 750 m), ocorreu maior concentração de indivíduos nas famílias Fabaceae, Euphorbiaceae, Combretaceae, Capparaceae e Nyctaginaceae. Juntas, representaram $76 \%$ do total de indivíduos do fragmento F4, 88\% de F5 e 91\% de F6.

A família Euphorbiaceae apresentou baixa densidade de indivíduos em F5, já a Nyctaginaceae, se destacou apenas na área F6. Por se tratar de área de transição, é muito comum encontrar na literatura áreas com essas características, classificadas como Caatinga ou Cerrado. Lemos e Meguro (2015) em uma área declivosa com altitude de $600 \mathrm{~m}$ encontraram comportamento parecido no táxon família, contudo, classificam a vegetação como Caatinga.

No nível superior de altitudes $(\geq 750 \mathrm{~m})$, ocorreu maior distribuição de indivíduos por famílias e maior variação entre áreas. No fragmento F7 (fitofisionomia Carrasco), apresentaram maiores números de indivíduos, em ordem decrescente, as famílias: Rutaceae, Nyctaginaceae, Euphorbiaceae, Fabaceae e Annonaceae, com 90\% de representatividade. Em F8 (Fitofisionomia de transição Carrasco Mata Úmida), se destacaram Fabaceae, Rutaceae, Euphorbiaceae e Malpighiaceae, concentrando 63\% do total de indivíduos. Já em F9 (Fitofisionomia de Mata 
Úmida), apresentaram maiores abundâncias, Lauraceae, Sapindaceae, Salicaceae, Fabaceae, Malpighiaceae e Ochnaceae, representando $77 \%$ do total de indivíduos.

As famílias com maiores concentrações de indivíduos tendem a variar com os estratos altitudes, sendo mais diversificadas nos níveis superiores.

Essa variação entre fragmentos é pouco perceptível pela análise de agrupamento, ao ser considerado a abundância de indivíduos por família. Com ponto de corte de 0,29 , foi formado um único grupo contendo oito fragmentos (F1, F2, F3, F4, F5, F6, F7 e F8), que apresentaram baixa similaridade com F9 (Figura 2).

Figura 2 - Dendrograma de agrupamento pelo método de grupo (UPGMA), com base no coeficiente de Morisita-Horn $\left(\mathrm{CH}_{\mathrm{i}}\right)$ e na variável família botânica, para nove fragmentos florestais distribuídos em três níveis de altitude na região do Araripe, Pernambuco, Brasil. Sendo: $C C C$, coeficiente de correlação cofenética; e $M$, ponto de corte conforme o índice de Mojena (1977)

Figure 2 - Group dendrogram by group method (UPGMA), based on the Morisita-Horn coefficient $\left(\mathrm{CH}_{i}\right)$ and the botanical family variable, for nine forest fragments distributed at three altitude levels in the Araripe region, Pernambuco state, Brazil. Being: CCC, co-behavioral correlation coefficient; and $M$, cut-off point according to the Mojena index (1977)

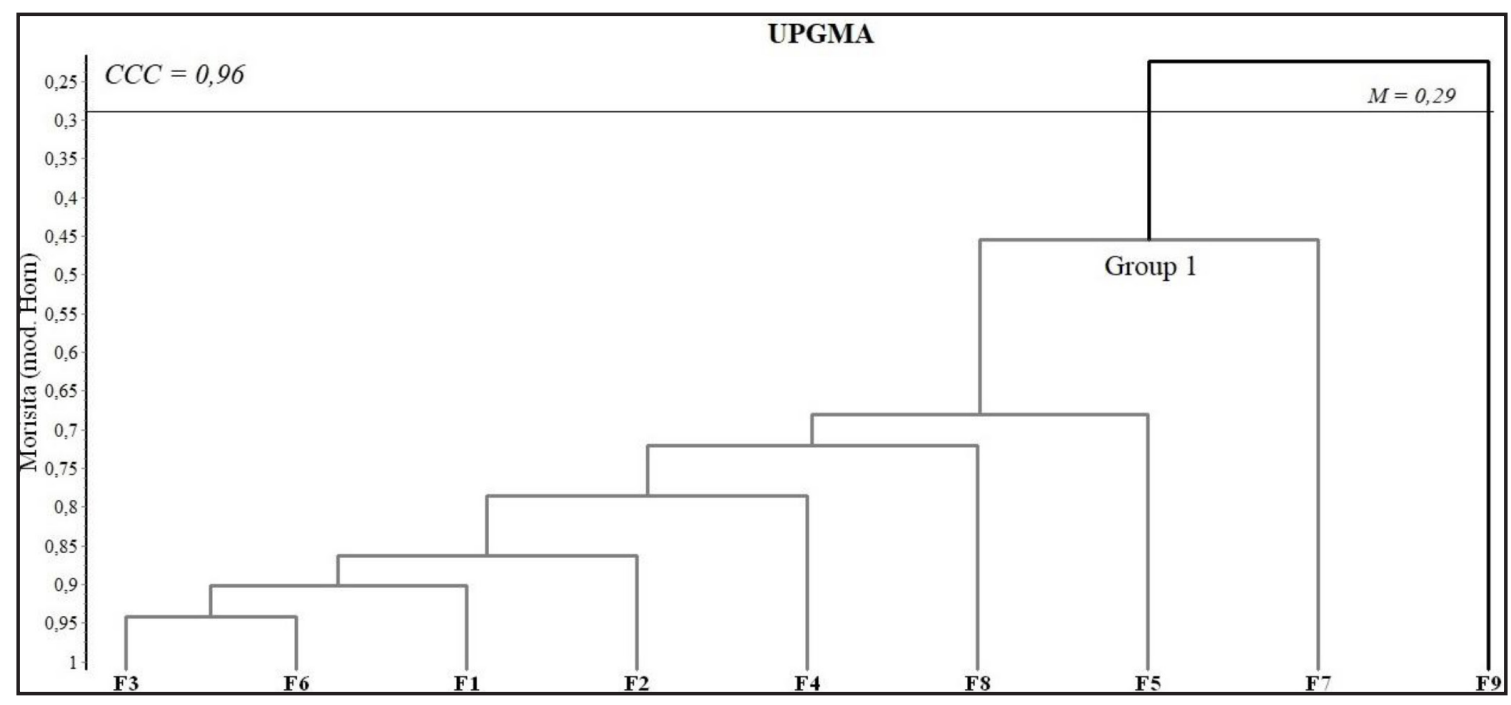

Fonte: Autores (2019)

Apesar de apresentar uma tendência de variação interna, é formado um único grupo, influenciado principalmente, pelo elevado número de indivíduos das famílias Fabaceae e Euphorbiaceae.

Em praticamente todas as formações vegetacionais, é comum se destacar a família Fabaceae, que apresenta elevada plasticidade, estando entre as mais ricas em número de espécies e indivíduos, já a Euphorbiaceae, se sobressai principalmente, em fitofisionomias encontradas nas Caatingas, sob clima semiárido, o que pode significar maior resistência e adaptação às severas condições ambientais, principalmente as secas prolongadas (AMORIM; SAMPAIO; ARAÚJO, 2005; BARBOSA et al., 2012; GUEDES et al., 2012; FERRAZ et al., 2013; HOLANDA et al., 2015; SANTOS; NASCIMENTO, 2017).

Nos fragmentos F2 e F3, também foi encontrado boa representatividade de indivíduos nas famílias Anacardiaceae, Apocynaceae e Rubiaceae, resultado similar aos encontrados por Apgaua et al. (2014) em Juvenília, Minas Gerais, e Farias et al. (2016), Serra talhada, Pernambuco, estudando vegetações de Caatinga.

Pela similaridade de Morisita-Horn, fica evidente a diferenciação do grupo de fragmentos 
localizados em regiões semiáridas (secas), com F9, única área de Floresta Úmida da amostragem. Podendo o clima, ser um influenciador nas semelhanças florísticas entre fragmentos.

Em F9, a baixa similaridade ocorreu principalmente, pela grande abundância de indivíduos nas famílias Lauraceae, Sapindaceae, Salicaceae, Malpighiaceae e Ochnaceae.

Para todas as análises das famílias botânicas, presença-ausência e abundância de indivíduos, foi verificado uma complexa variação da composição florística, apresentando: os fragmentos do nível inferior de altitude, maiores similaridades entre si e menores com os do estrato superior; os do estrato intermediário, se assemelhando com os níveis inferior e superior; e as áreas do estrato superior de altitudes com menores semelhanças entre si e com os demais fragmentos. F9, nas duas situações, apresentou composição de famílias distinta de todas as áreas.

\section{Riqueza e similaridade dos fragmentos florestais pela distribuição de espécies lenhosas}

Das 153 espécies amostradas na região, 66 pertencem ao nível inferior de altitude, 65 ao intermediário e 83 no estrato superior. Dessas, apenas 13 (8\%) ocorrem simultaneamente nos três níveis de altitudes, 32 (21\%) com exclusividade no estrato inferior, 19 (12\%) no intermediário e 52 do superior.

No intervalo intermediário de altitude ocorreu maior compartilhamento de espécies com os outros dois estratos. Já entre os níveis inferior e superior, há menor reciprocidade de espécies, com apenas três ( $2 \%)$.

Levando em consideração a presença-ausência de espécies, pela da curva de rarefação através do intervalo de confiança, com $95 \%$ de probabilidade, foi verificado os três níveis de altitudes com possibilidade de semelhanças na riqueza de espécies (Figura 3).

Figura 3 - Curva de rarefação, com intervalo de confiança de $95 \%$ de probabilidade, do número espécies ocorrentes em três níveis de altitudes na região do Araripe, Pernambuco, Brasil

Figure 3 - Rarefaction curve of the number of species occurring at three levels of altitude in the Araripe region, Pernambuco state, Brazil (with 95\% confidence interval)

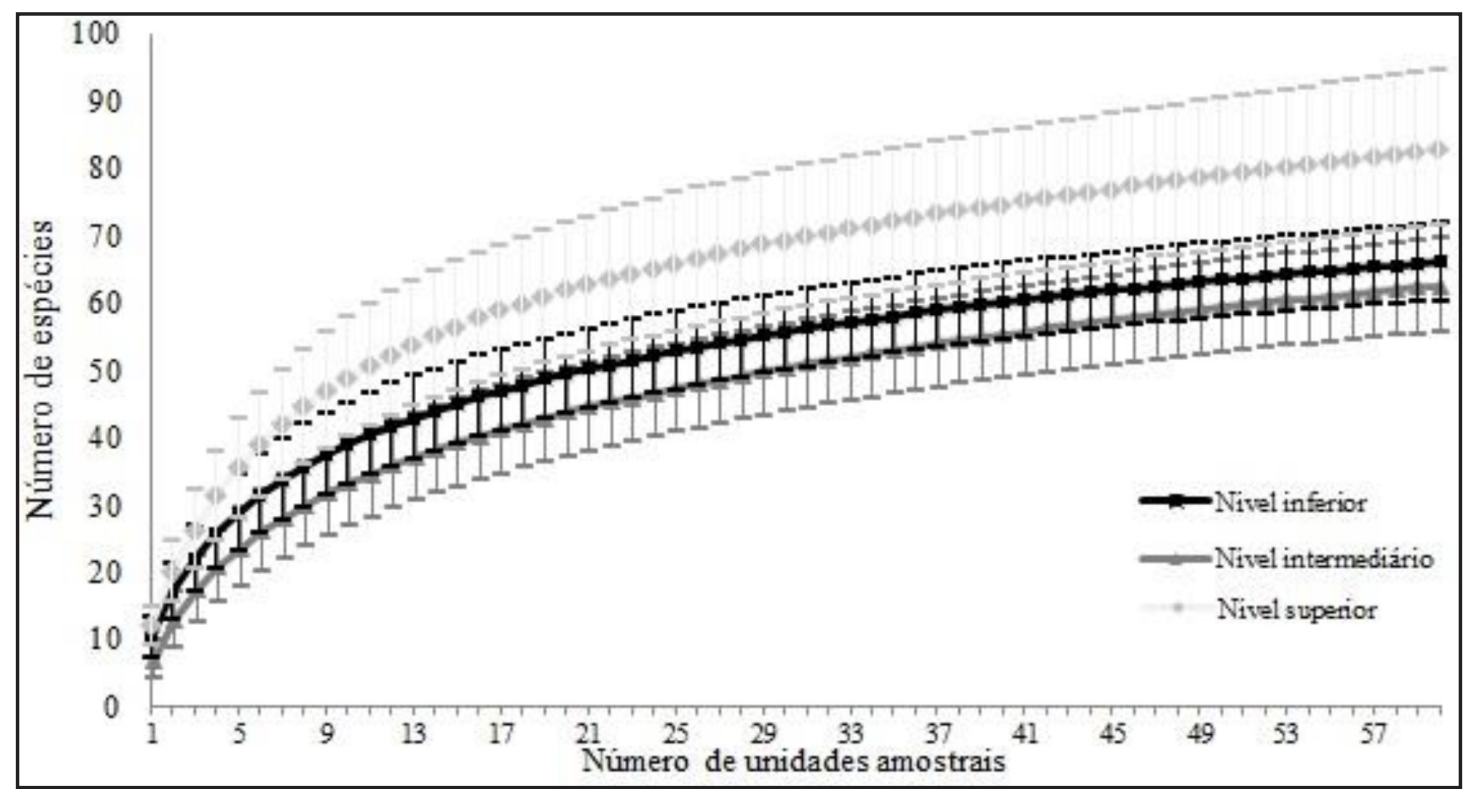

Fonte: Autores (2019) 
Estatisticamente, pelo intervalo de confiança, pode ser encontrado: no nível inferior de altitude, de 60 a 72 espécies; no intermediário, de 58 a 72; e no superior, de 71 a 95. Apesar dos ambientes tenderem a semelhança, é observado uma maior variabilidade de espécies por unidade amostral em níveis superiores de altitudes, com erro de 12.

Entre fragmentos, no estrato inferior de altitude, a área F2 apresentou maior diversidade de espécies $(51 \pm 6)$ que F1 $(35 \pm 6)$ e F3 $(27 \pm 8)$, que por sua vez podem se assemelhar estatisticamente. A semelhança entre as áreas F1 e F3 pode ser justificada pela mesma fitofisionomia (Caatinga ArbustivaArbórea), contudo, F3 apresentou maior variabilidade de espécies entre parcelas, com erro de \pm 8 , podendo estar associado à menor idade do fragmento, que apresenta aproximadamente 25 anos.

No nível intermediário de altitude, o fragmento F4 apresentou maior riqueza de espécies, com 43 ( \pm 6$)$. Já F5 e F6 podem se assemelhar estatisticamente, com $28( \pm 4)$ e $30( \pm 6)$ espécies. Todas as áreas desse estrato apresentaram baixa variação estatística. A maior diversidade de F4 pode estar associada à maior umidade do fragmento, visto que, está localizado próximo de um reservatório de água e com vários cursos intermitentes em seu interior.

No estrato superior de altitude, a maior riqueza de espécies foi encontrada no fragmento F8 $(41 \pm 7)$

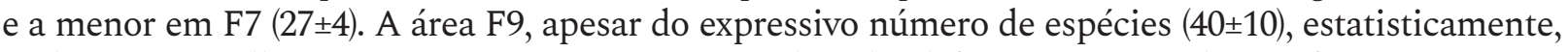
pode se assemelhar com F7 e F8. Nesse estrato altitudinal foram encontrados os fragmentos com maiores variabilidades, principalmente, das áreas F8 e F9.

Os fragmentos com maiores proximidades a cursos d'água (F2, F4, F8 e F9), além de apresentaram maiores diversidade de espécies, tenderam a se assemelharem estatisticamente.

Pelas curvas de rarefação e intervalos de confiança, foi verificada uma possível semelhança entre estratos de altitudes, principalmente, pela elevada variabilidade dos fragmentos, que aumentou o erro da média e consequentemente, os intervalos de confiança. Foi verificado maiores diferenças estatísticas, entre fragmentos, com tendência de a riqueza aumentar naqueles com maiores proximidades a fontes de umidade.

A variação na riqueza de espécies entre fragmentos fica mais evidente quando é considerada a densidade de indivíduos. O índice de Shannon, além do número de espécies leva em consideração o padrão de distribuição de indivíduos. Na Tabela 2 , são observadas, pelo teste $t$ com $95 \%$ de probabilidade, maiores diferenças estatísticas na diversidade entre áreas.

\section{Tabela 2 - Riqueza de espécies lenhosas pelo índice de Shannon ('H) e equabilidade de Pielou $(J)$ de fragmentos distribuídos em três níveis de altitudes na região do Araripe, Pernambuco, Brasil}

Table 2 - Richness of woody species by Shannon index ('H) and Pielou equability (J) of fragments distributed at three levels of altitudes in the Araripe region, Pernambuco state, Brazil

\begin{tabular}{|c|c|c|c|c|c|}
\hline Níveis & Fragmentos & Número de espécies & Número de indivíduos & Shannon $H^{\prime}$ & Pielou $J$ \\
\hline \multicolumn{6}{|c|}{ Inferior (altitudes $\leq 600 \mathrm{~m}$ ) } \\
\hline & $\mathrm{F} 1$ & 35 & 436,0 & $2,45_{\mathrm{e}}$ & 0,7 \\
\hline & $\mathrm{F} 2$ & 51 & 1.068 & $3,19{ }_{a}$ & 0,8 \\
\hline & F3 & 27 & 553,0 & $2,19 \mathrm{~g}$ & 0,7 \\
\hline \multicolumn{6}{|c|}{ Intermediário $(600<$ altitudes $<750 \mathrm{~m})$} \\
\hline & $\mathrm{F} 4$ & 43 & 1.032 & $2,49_{\mathrm{de}}$ & 0,7 \\
\hline & F5 & 28 & 844,0 & $2,33_{\mathrm{f}}$ & 0,7 \\
\hline & F6 & 30 & 657,0 & $2,42_{\mathrm{e}}$ & 0,7 \\
\hline & & & & \multicolumn{2}{|c|}{$\begin{array}{l}\text { Continua ... } \\
\text { Continuation }\end{array}$} \\
\hline
\end{tabular}


Tabela 2 - Conclusão ...

Table 2 - Conclusion ...

\begin{tabular}{|c|c|c|c|c|c|}
\hline Níveis & Fragmentos & Número de espécies & Número de indivíduos & Shannon $H^{\prime}$ & Pielou $J$ \\
\hline \multicolumn{6}{|c|}{ Superior (altitudes $\geq 750 \mathrm{~m}$ ) } \\
\hline & F7 & 27 & 644,0 & $2,30_{f}$ & 0,7 \\
\hline & F8 & 41 & 707,0 & $3,10_{b}$ & 0,8 \\
\hline & F9 & 40 & 1.046 & $2,88_{c}$ & 0,8 \\
\hline
\end{tabular}

Fonte: Autores (2019)

$\mathrm{O}$ peso da distribuição de indivíduos entre espécies fica evidente ao analisar a área $\mathrm{F} 4$, que mesmo obtendo a segunda maior riqueza (43), obteve apenas a quarta posição no ranking do índice de diversidade de Shannon. Esse fragmento apresentou $68 \%$ de toda densidade de indivíduos concentrada em quatro espécies (Croton blanchetianus Baill., Combretum glaucocarpum Mart., Dalbergia frutescens Vell. Britton e Myrcia guianensis Aubl. DC.) e 70\% das espécies representadas por menos de 10 indivíduos.

No nível inferior de altitude, o fragmento F2 apresentou maior diversidade pelo índice de Shannon. Já os fragmentos F1 e F3, diferentemente do que ocorre pela análise da curva de rarefação (presença-ausência de espécies), não se assemelharam estatisticamente, apresentando a área F3 o menor $H^{\prime}$.

A maior riqueza de F2 nesse estrato de altitude, pode ser justificada por se tratar de um fragmento com fitofisionomia Caatinga Arbórea, aparentemente com maior umidade, vegetação densa, rica em arbustos e estágios de sucessão mais definidos.

Já os fragmentos F1 e F3, apesar de possuírem a mesma fitofisionomia (Caatinga Arbustivoarbórea), em F3 foi encontrado menor diversidade, o que pode ser reflexo do seu tempo de existência, por ser mais jovem (aproximadamente, 25 anos), ou até mesmo pelas características da área, que apresenta solos rasos e afloramentos rochosos. Na literatura é verificado que a disponibilidade hídrica, idade do fragmento e fitofisionomia local são os principais responsáveis pela variação da diversidade das Caatingas (RODAL; MARTINS; SAMPAIO, 2008; HOLANDA et al., 2015; FARIAS et al., 2016).

No nível intermediário de altitude, os fragmentos apresentaram maiores semelhanças estatísticas, dentro e entre os demais estratos (inferior e superior).

No estrato superior de altitude, as áreas F8 e F9 apresentaram elevados índices de diversidade, com a segunda e terceira posição no ranking de $H$ '. Já F7, apresentou baixa diversidade, se assemelhando à área F5 do nível intermediário, podendo ser justificado por serem áreas muito próximas e apresentarem praticamente uma continuidade vegetacional.

De maneira geral, pelo índice de Shannon é verificada uma tendência de fragmentos florestais com menores altitudes, apresentarem riquezas de espécies inferiores, com exceção de F2, que por condições ambientais atípicas (umidade) apresentou elevada diversidade. O nível intermediário de altitude também apresentou baixa diversidade, se assemelhando a fragmentos dos níveis inferior e superior, podendo ser considerado como ambiente de transição. Já no nível superior de altitude, foi encontrada maior diversidade florística.

Neves et al. (2016) encontraram resultado semelhante na Chapada Diamantina, Bahia, em que, estudando composições florísticas do cerrado, caatinga e área de encosta, também encontrou fitofisionomias variadas, com maior riqueza de espécies em altitudes superiores.

Pela equabilidade de Pielou, todas as áreas mostraram bons padrões de distribuição de indivíduos entre espécies, sendo mais evidenciado nas áreas F2, F8 e F9.

Analisando a similaridade florística entre fragmentos, pela presença-ausência de espécies, pode ser verificado através da análise de agrupamento, índice de Sorenson pelo método UPGMA e com ponto de corte de 0,43 , a formação três grupos (Figura 4). 
Figura 4 - Dendrograma de agrupamento pelo método de grupo (UPGMA), com base no coeficiente de Sorenson $\left(\mathrm{SO}_{i}\right)$ e na variável espécie lenhosas, para os fragmentos florestais distribuídos em três níveis de altitudes, na região do Araripe, Pernambuco, Brasil. Sendo: $C C C$, coeficiente de correlação cofenética; $M$, ponto de corte conforme o índice de Mojena (1977)

Figure 4 - Group Dendrogram by group method (UPGMA), based on the Sorenson coefficient $\left(\mathrm{SO}_{i}\right)$ and the woody species variable for the forest fragments distributed in three altitude levels, in the region of Araripe, Pernambuco state, Brazil. Being: CCC, co-behavioral correlation coefficient; $M$, cut-off point according to the Mojena index (1977)

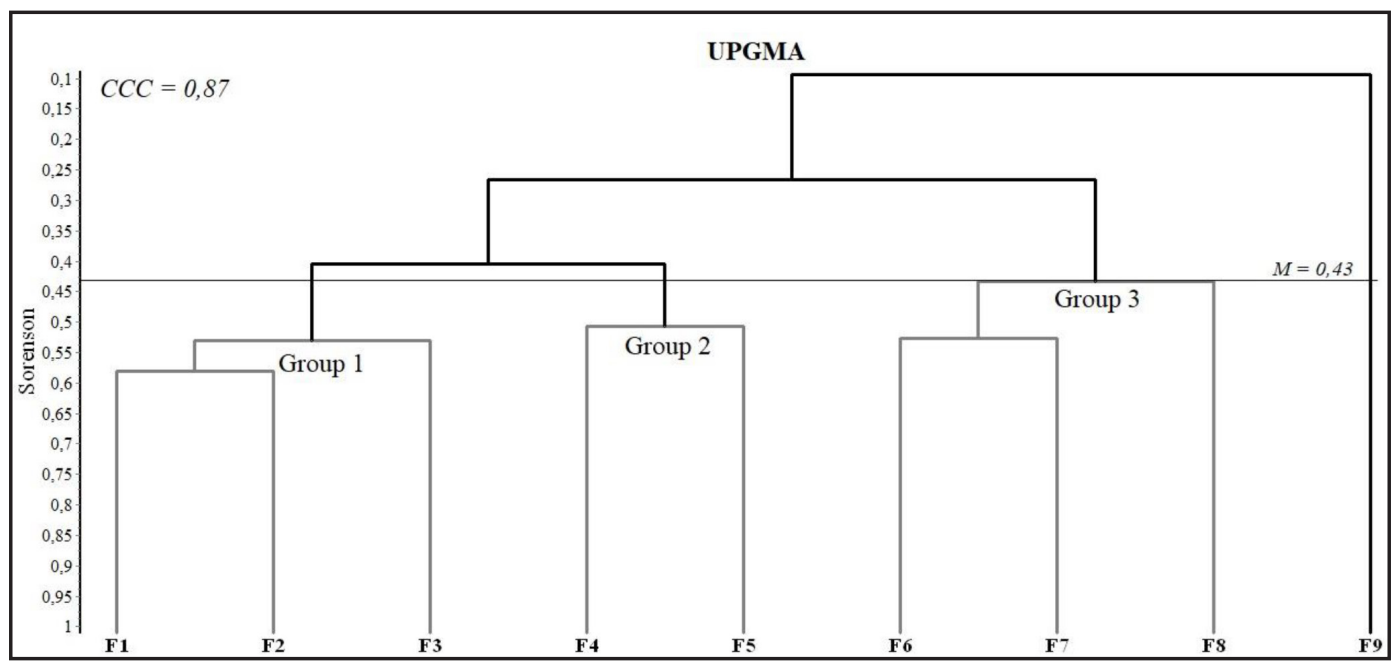

Fonte: Autores (2019)

O primeiro grupo, com $52 \%$ de similaridade, foi formado exclusivamente, por fragmentos do nível inferior de altitude ( $\leq 600 \mathrm{~m})$. As áreas F1, F2 e F3 compartilharam 15 espécies lenhosas (Allophylus quercifolius Radlk., Anadenanthera colubrina (Vell.) Brenan, Annonaleptopetala (R.E. Fr.) H. Rainer, Bauhinia subclavata Benth., Commiphora leptophloeos (Mart.) J.B. Gillett, Croton blanchetianus, Cynophalla flexuosa (L.) J. Presl., Guapira opposita (Vell.) Reitz, Jatropha mollissima (Pohl) Baill., Myracrodruon urundeuva Allemão, Piptadenia viridiflora (Kunth) Benth., Poincianella gardneriana (Benth.) L.P. Queiroz, Sapium glandulosum (L.) Morong, Schinopsis brasiliensis Engl. e Xylosma prockia (Turcz.) Turcz).

O segundo grupo, com $50 \%$ de similaridade, écomposto pelas áreas F4e F5, do nível intermediário de altitude (de 600 a $750 \mathrm{~m}$ ), apresentando 17 espécies comuns (Annona leptopetala, Bauhinia subclavata, Chloroleucon foliolosum (Benth.) G.P. Lewis, Colicodendron yco Mart., Combretum glaucocarpum, Croton limae A.P.S. Gomes, M.F. Sales \& P.E. Berry, Cynophalla flexuosa, Dalbergia frutescens, Guapira opposita, Handroanthus impetiginosus, Myrcia guianensis, Piptadenia viridiflora, Sapium glandulosum, Schinopsis brasiliensis, Senegalia langsdorffii (Benth.) Seigler \& Ebinger, Senegalia polyphylla (DC.) Britton \& Rose, e Senna splendida (Vogel) H.S.Irwin \& Barneby).

Os dois primeiros grupos apresentaram similaridade de aproximadamente $40 \%$, com oito espécies com ocorrência simultânea nos cinco fragmentos (Allophylus quercifolius, Annona leptopetala, Bauhinia subclavata, Cynophalla flexuosa, Guapira opposita, Piptadenia viridiflora, Sapium glandulosum e Schinopsis brasiliensis).

O grupo três, foi formado pelos fragmentos F6, F7 e F8, áreas com altitudes superiores a $700 \mathrm{~m}$ e localizadas sob clima semiárido, compartilhando 10 espécies lenhosas (Annona leptopetala, Bauhinia acuruana, Byrsonima gardneriana A. Juss., Combretum glaucocarpum, Croton limae, Eugenia flavescens DC., Guapira opposita, Pilocarpus spicatus subsp. aracatensis Kaastra, Senegalia langsdorffii e Swartzia psilonema 
Harms).

Da mesma forma que ocorreu na análise de famílias botânicas, a área F9 apresentou baixa similaridade florística com os demais fragmentos, com 31 espécies exclusivas, que representam $78 \%$ da riqueza do fragmento.

Entre níveis de altitudes, o inferior apresentou maior similaridade entre fragmentos (entre 50 e 60\%), com as três áreas se agrupando por apresentarem elevado número de espécies comuns. No intervalo intermediário, apesar possuir de nove espécies comuns entre áreas (Annona leptopetala, Bauhinia subclavata, Colicodendron yco, Combretum glaucocarpum, Croton limae, Cynophalla flexuosa, Dalbergia frutescens, Guapira opposita e Senegalia langsdorffii), apenas as áreas F4 e F5 se agruparam. O fragmento F6 se agrupou com F7 do nível superior.

No nível superior de altitude, apenas quatro espécies ocorreram comumente nos três fragmentos (Erythroxylum caatingae Plowman, Guapira opposita, Poeppigia procera C. Presl. e Swartzia psilonema), representando maior heterogeneidade florística entre áreas.

A Guapira opposita foi a única espécie com distribuição em todos os fragmentos estudados. $\mathrm{Na}$ literatura há registro de ocorrência desta espécie em todo território nacional, nas mais variadas fitofisionomias (OLIVEIRA et al., 2012; ALVES et al., 2015; FERNANDES; QUEIROZ, 2015), podendo ser altamente recomendada em projetos de recuperação de áreas degradas em toda região do Araripe, independentemente do gradiente topográfico. A Annona leptopetala esteve presente em oito fragmentos (exceto o F9), Bauhinia subclavata e Combretum glaucocarpum em sete, estando estas representadas em todos níveis de altitude.

Considerando a abundância de indivíduos por espécie, pela análise de agrupamento, elaborado pelo índice de Morisita-Horn $\left(\mathrm{CH}_{\mathrm{I}}\right)$, método UPGMA e ponto de corte de 0,32, foram formados dois grupos (Figura 5).

Figura 5 - Dendrograma de agrupamento pelo método de grupo (UPGMA), com base no coeficiente de Morisita-Horn $\left(\mathrm{CH}_{i}\right)$ e na variável espécie lenhosa, para os fragmentos florestais distribuídos em três níveis de altitudes, na região do Araripe, Pernambuco, Brasil. Sendo: $C C C$, coeficiente de correlação cofenética; $M$, ponto de corte conforme o índice de Mojena (1977)

Figure 5 - Group dendrogram by group method (UPGMA), based on Morisita-Horn coefficient

$\left(\mathrm{CH}_{i}\right)$ and woody species variable, for the forest fragments distributed at three altitudes

levels, in the Araripe region, Pernambuco state, Brazil. Being: CCC, co-behavioral correlation coefficient; $M$, cut-off point according to the Mojena index (1977)

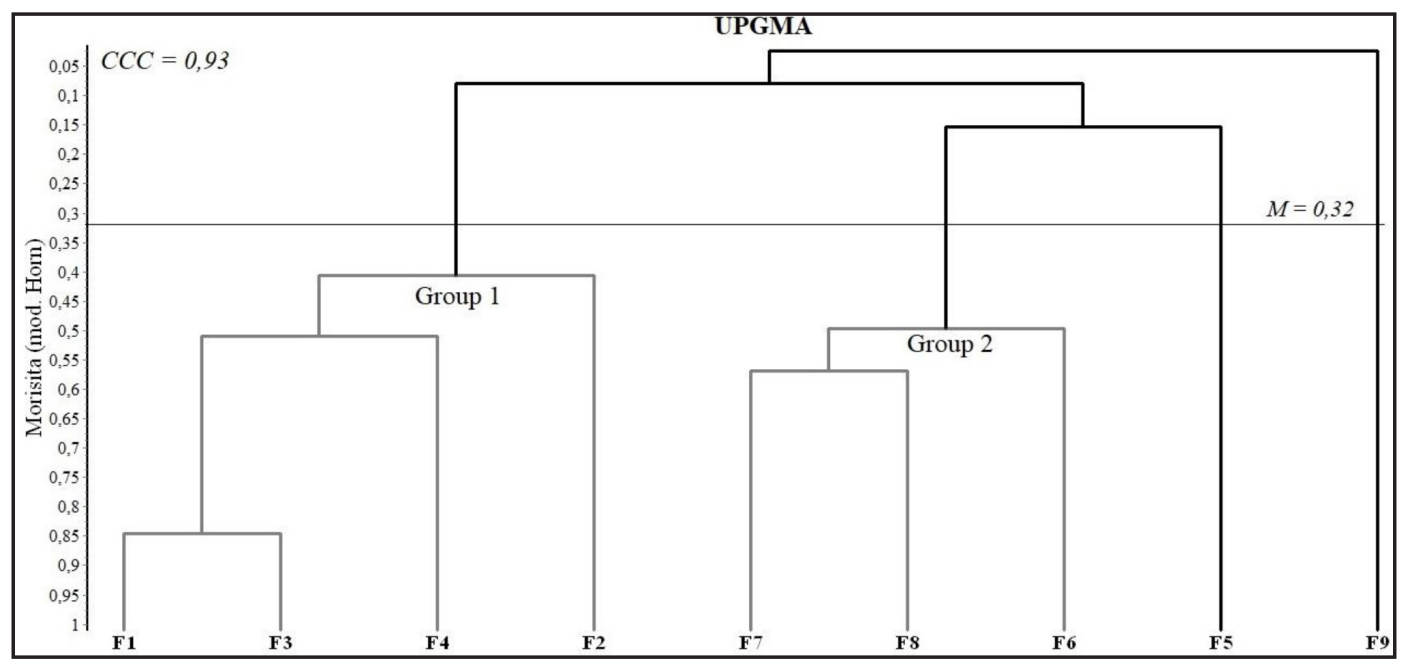

Fonte: Autores (2019) 
O primeiro grupo, com similaridade de $40 \%$, foi composto pelos fragmentos F1, F2, F3 e F4, sendo três do nível inferior de altitude e um do intermediário. A formação desse grupo se deve principalmente, a espécie Croton blanchetianus, que apresenta elevada abundância nos quatro fragmentos. As áreas F1 e F3 apresentaram aproximadamente, 85\% de semelhança, que além do Croton blanchetianus, compartilham muitos indivíduos da Poincianella gardneriana. Essas espécies, conhecidas popularmente como marmeleiro-preto e catingueira são encontradas em abundância em diversos trabalhos realizados nas fitofisionomias das Caatingas (BARBOSA et al., 2012; GUEDES et al., 2012; FERRAZ et al., 2013; APGAUA et al., 2014; FARIAS et al., 2016).

O segundo grupo, com similaridade de $45 \%$, foi formado pelos fragmentos F6, F7 e F8, todos com altitudes superiores a $700 \mathrm{~m}$, sendo um do nível intermediário e dois do nível superior. As espécies comumente abundantes foram Croton limae e Guapira opposita.

As áreas F5 e F9, apresentaram as espécies mais abundantes distintas dos demais fragmentos, apresentando baixas similaridades. No fragmento F5, obtiveram maiores abundâncias de indivíduos: Combretum glaucocarpum, Cynophalla flexuosa e Senegalia polyphylla. Em F9: Ocotea nitida (Meisn.) Rohwer, e Matayba guianensis Aubl..

Algumas espécies, apesar da elevada densidade de indivíduos são encontradas restritas a um estrato de altitude ou fragmento.

No nível inferior de altitude, entre as 10 espécies com maiores números de indivíduos, cinco (Poincianella gardneriana, Parapiptadenia zehntneri (Harms) M.P. Lima \& H.C. Lima, Coutarea alba Griseb., Aspidosperma cuspa (Kunth) S.F. Blake ex Pittier e Amburana cearensis (Allemão) A.C. Sm.) são exclusivas desse estrato, sendo duas (Coutarea alba e Aspidosperma cuspa) ocorrentes apenas na área F2.

Croton blanchetianus, a espécie mais ocorrente do nível inferior de altitude, também se destaca na área F4 do nível intermediário. Piptadenia viridiflora ocorre em todos níveis, sendo mais abundante no inferior e intermediário.

No estrato superior de altitude, entre as 10 espécies com maior abundância de indivíduos, seis (Ocotea nítida, Matayba guianensis, Metrodorea mollis Taub., Byrsonima vacciniifolia A. Juss., Ouratea parviflora (A.DC.) Baill., e Casearia grandiflora Cambess) apresentaram ocorrência restrita ao estrato, sendo quatro (Ocotea nítida, Matayba guianensis, Ouratea parviflora e Casearia grandiflora) exclusivas do fragmento F9.

No intervalo intermediário de altitude, entre as 10 espécies com maior densidade de indivíduos, apenas Pityrocarpa moniliformis apresentou exclusividade ao estrato e a área F6.

De maneira geral, os estratos de altitudes podem ser considerados importantes influenciadores na variação florística, apresentando os níveis inferior e superior, composições com características próprias, com muitas espécies exclusivas, e o nível intermediário funcionando como ecótono, com menos espécies exclusivas e alta similaridade com os outros dois níveis de altitudes.

As variações nos fragmentos do nível superior de altitude tendem a acompanhar os gradientes de umidade, apresentando a parte mais seca (fronteira com o estado de Piauí), vegetação de menor porte e composição menos diversificada, e maior riqueza de espécies nas regiões mais úmidas (fronteira com o estado do Ceará).

Amostragens florestais com estratificação de altitudes são extremamente importantes, podendo trazer respostas úteis para fins ecológicos e econômicos, sendo essenciais na elaboração de planos de manejo mais precisos, visto que, a ocorrência e abundância de espécies tendem a variar com o gradiente topográfico. Segundo Martins et al. (2003), o conhecimento sobre a variação da vegetação ao longo de gradientes topográficos é essencial na estratégia de conservação e manejo dos remanescentes florestais, bem como na restauração e recuperação de áreas degradadas. 


\section{Conclusões}

Pelas variações verificadas entre e dentro dos estratos de altitudes, pode ser inferido que a região do Araripe em Pernambuco, apresenta uma enorme diversidade florística, abrigando um verdadeiro mosaico de diferentes fitofisionomias, com composições e estruturas que tendem a diversificar em conformidade aos efeitos orográficos.

Em altitudes de até $600 \mathrm{~m}$, a vegetação predominante pertence à fitofisionomia das Caatingas, com características próprias e elevada similaridade florística entre fragmentos. Entre 600 e 750 m, estão localizadas áreas de transição, ditas como ecótono ecológico, apresentando poucas espécies exclusivas e muitas compartilhadas com os níveis inferior e superior. Na chapada, em altitudes superiores a $750 \mathrm{~m}$, ocorre maior heterogeneidade vegetacional, com riqueza e porte dos indivíduos lenhosos variando em função ao gradiente de umidade.

A variação florística encontrada nesse trabalho, corrobora a importância da estratificação altitudinal e replicação de fragmentos florestais em estudos científicos, visto que, em pequenas distâncias geográficas ocorreram discrepantes variações na ocorrência e abundância das espécies.

\section{Agradecimentos}

O presente trabalho foi realizado com apoio da Coordenação de Aperfeiçoamento de Pessoal de Nível Superior - Brasil (CAPES), código de financiamento 001 e Fundação de Amparo à Ciência e Tecnologia do Estado de Pernambuco (FACEPE).

\section{Referências}

ALVARES, C. A. et al. Köppen's climate classification map for Brazil. Meteorologische Zeitschrift, Stuttgard, v. 22, n. 6, p. 711-728, 2013.

ALVES, M. et al. Levantamento florístico de um remanescente de Mata Atlântica no litoral norte do estado da Bahia, Brasil Maria. Hoehnea, São Paulo, v. 42, n. 3, p. 581-595, 2015.

AMJAD, M. S. et al. Floristic composition, biological spectrum and conservation status of the vegetation in Nikyal valley, Azad Jammu and Kashmir. Asian Pacific Journal of Tropical Disease, [s. l.], v. 6, n. 1, p. 63-69, 2016.

AMORIM, I. L.; SAMPAIO, E. V. S. B.; ARAÚJO, E. L. Flora e estrutura da vegetação arbustivo-arbórea de uma área de caatinga do Seridó, RN, Brasil. Acta Botânica Brasílica, Belo Horizonte, v. 19, n. 3, p. 615-623, 2005.

APGAUA, D. M. G. et al. Tree community structure in a seasonally dry tropical Forest remnant, Brazil. Cerne, Lavras, v. 20, n. 2, p. 173-182, 2014.

ARAÚJO, F. S. et al. Floristics and life-forms along a topographic gradient, central-western Ceará, Brazil. Rodriguésia, Rio de Janeiro, v. 62, n. 2, p. 341-366, 2011.

ARAÚJO, S. M. S. A região semiárida do Nordeste do Brasil: questões ambientais e possibilidades de uso sustentável dos recursos. Revista Rios Eletrônica, Paulo Afonso, v. 5, n. 5, p. 89-98, 2011.

ARRUDA, D. M. et al. Landforms and soil attributes determine the vegetation structure in the Brazilian semiarid. Folia Geobotanica, Praha, 50, p. 175-184, 2015.

BARBOSA, M. D. et al. Florística e fitossociologia de espécies arbóreas e arbustivas em uma área de Caatinga em Arcoverde, PE, Brasil. Revista Árvore, Viçosa, MG, v. 36, n. 5, p. 851-858, 2012.

BRASIL. Ministério do Meio Ambiente. Potencial Florestal da Região do Araripe. In: REGIÃO do Araripe, Pernambuco, Diagnóstico Florestal. Brasília, 2007. p. 15-36. 
COLWELL, R. K. EstimateS: statistical estimation of species richness and shared species from samples. Version 9. User's Guide and application. [S. l.: s. n.], 2013. Disponível em: http://purl.oclc.org/ estimates. Acesso em: 12 ago. 2018.

FARIAS, S. G. G. et al. Fisionomia e estrutura de vegetação de caatinga em diferentes ambientes em Serra Talhada - Pernambuco. Ciência Florestal, Santa Maria, v. 26, n. 2, p. 435-448, 2016.

FERNANDES, M. F.; QUEIROZ, L. P. Floristic surveys of Restinga Forests in southern Bahia, Brazil, reveal the effects of geography on community composition. Rodriguésia, Rio de Janeiro, v. 66, n. 1, p. $51-73,2015$.

FERRAZ, R. C. et al. Levantamento fitossociológico em área de Caatinga no Monumento Natural Grota do Angico, Sergipe, Brasil. Revista Caatinga, Mossoró, v. 26, n. 3, p. 89-98, 2013.

GADELHA, F. H. L. et al. Produtividade de clones de eucaliptos em diferentes sistemas de manejo para fins energéticos. Pesquisa Florestal Brasileira, Colombo, v. 35, n. 83, p. 263-270, 2015.

GUEDES, R. S. et al. Caracterização florístico-fitossociológica do componente lenhoso de um trecho de caatinga no semiárido paraibano. Revista Caatinga, Mossoró, v. 25, n. 2, p. 99-108, 2012.

HAMMER, O.; HARPER, D. A. T.; RYAN, P. D. PAST: Paleontological Statistic software package for education and data analysis. Paleontologia Eletronica, [s. l.], v. 4, n. 1, p. 1-9, 2001. Disponível em: http://palaeoelectronica.org/2001_1/past/issue1_01.htm. Acesso em: 04 ago. 2018.

HIGUCHI, P. et al. Florística e estrutura do componente arbóreo e relação com variáveis ambientais em um remanescente florestal em Campos Novos - SC. Ciência Florestal, Santa Maria, v. 26, n. 1, p. $35-46,2016$.

HOLANDA, A. C. et al. Estrutura da vegetação em remanescentes de caatinga com diferentes históricos de perturbação em Cajazeirinhas (PB). Revista Caatinga, Mossoró, v. 28, n. 4, p. 142-150, 2015.

IBGE. Manual técnico da vegetação brasileira. 2. ed. rev. e ampl. Rio de Janeiro, 2012. 271 p.

LEMOS, J. R.; MEGURO, M. Estudo fitossociológico de uma área de Caatinga na Estação Ecológica (ESEC) de Aiuaba, Ceará, Brasil. Biotemas, Florianopolis, v. 28, n. 2, p. 39-50, 2015.

MARTINS, S. V. et al. Distribuição de espécies arbóreas em um gradiente topográfico de Floresta Estacional Semidecidual em Viçosa, MG. Scientia Forestalis, Piracicaba, n. 64, p. 172-181, 2003.

MOJENA, R. Hierarchical grouping methods and stopping rules: an evaluation. The Computer Journal, [s. l.], v. 20, n. 4, p. 359-363, 1977.

NEVES, S. P. S. et al. What are the most important factors determining different vegetation types in the Chapada Diamantina, Brazil? Brazilian Journal of Biology, São Carlos, v. 76, n. 2, p. 315-333, 2016.

OLIVEIRA, L. S. B. et al. Structure of the woody component of an Atlantic forest Fragment, Moreno PE. Semina: Ciências Agrárias, Londrina, v. 33, n. 1, p. 203-212, 2012.

RODAL, M. J. N.; MARTINS, F. R.; SAMPAIO, E. V. S. B. Levantamento quantitativo das plantas lenhosas em trechos de vegetação de Caatinga em Pernambuco. Caatinga, Mossoró, v. 21, n. 3, p. 192205, 2008.

SANTANA, J. A. S. et al. Estrutura e distribuição espacial da vegetação da Caatinga na Estação Ecológica do Seridó, RN. Pesquisa Florestal Brasileira, Colombo, v. 36, n. 88, p. 355-361, 2016.

SANTOS, F. L. A.; NASCIMENTO, F. R. Dinâmica hidroclimática do Planalto da Ibiapaba e sua depressão periférica circunjacente: estudo de caso nos municípios de Tianguá e Ubajara-Noroeste do Ceará. Revista Ra'e Ga, Curitiba, v. 39, p. 57-75, 2017.

SILVA, J. O. et al. Floristic composition and phytogeography contextualization of the natural regeneration of an Alluvial Forest located in the "Planalto Sul Catarinense" Region, SC, Brazil. Revista Árvore, Viçosa, MG, v. 41, n. 2, p. 1-9, 2017. 